J. DIFFERENTIAL GEOMETRY

11 (1976) 299-308

\title{
EXISTENCE OF INFINITELY MANY CLOSED GEODESICS
}

\author{
WILHELM KLINGENBERG
}

In memory of Yôtarô Tsukamoto

Let $M$ be a compact simply connected differentiable manifold. By $(M, g)$ we denote $M$ endowed with a riemannian metric $g$. We ask whether the riemannian manifold $(M, g)$ possesses an infinite number of prime closed geodesics, i.e., closed geodesics which are not the covering of another closed geodesic. Gromoll and Meyer [6] have shown that the answer to our question is affirmative if the rational homotopy type of $M$ has a certain degree of complicateness, i.e., if, as a first approximation, $M$ must have the rational homotopy type of a product. On the other hand, the question is still open, for instance, for manifolds of the rational homotopy type of a sphere or one of the other symmetric spaces of rank 1 .

In this note we will show that, at least generically, the answer to our question is affirmative for any manifold. More precisely, we will show the existence of a residual subset $\mathscr{G}^{*}$ in the space $\mathscr{G}=\mathscr{G}(M)$ of riemannian metrics on $M$ such that for any $g^{*} \in \mathscr{G}^{*}$ the riemannian manifold $\left(M, g^{*}\right)$ has an infinite number of prime closed geodesics. Essential tools in our proof will be, on the one hand, a result of D. Sullivan [11] on the rational homology of the space $\Lambda M$ of closed curves on $M$ and, on the other hand, a result of Eliasson [4] on the structure of the negative gradient flow of the energy integral $E$ on $\Lambda M$. In our main result we clearly can replace the hypothesis that the fundamental group $\pi_{1} M$ is zero by the weaker hypothesis that $\pi_{1} M$ is finite.

1. Let $\mathscr{G}=\mathscr{G}(M)$ be the Frechet space of $C^{\infty}$ riemannian metrics on $M$ with the $C^{\infty}$-metric. For a given $g \in \mathscr{G}$ and each nontrivial closed geodesic $c$ on $(M, g)$ we consider the Poincaré map $P_{c}$ associated with $c$ (cf. [8], [10]). Since $P_{c}$ is a symplectic map, we can write its eigenvalues $\rho$ with $|\rho|=1$ in the form

$$
e^{ \pm 2 \pi i a}, \quad 0 \leq a \leq \frac{1}{2} .
$$

Let $A(M, g)$ be the set of these numbers $a$ for all closed geodesics on $(M, g)$. We are interested in the following property:

Communicated September 13, 1974. The subject of this paper is a project of the "Sonderforschungsbereich Theoretische Mathematik" at University of Bonn. 
( $\alpha$ ) The set $A(M, g) \cup\{1\}$ is free over the rationals.

In other words, the property $(\alpha)$ means that none of the $a \in A(M, g)$ is rational and any finite set of elements of $A(M, g)$ is independent of the rationals.

1.1. Lemma. The property $(\alpha)$ is residual on $\mathscr{G}(M)$.

Proof. For each integer $m>0$ we consider the property of $A=A(M, g)$ :

$(\alpha, m)$ Given any family $\left\{k_{a}, a \in A\right\}$ of integers $k_{a}$ satisfying $\sum\left|k_{a}\right|=m$, one has $\sum_{a \in A} k_{a} a \notin Z$.

Note that $(\alpha, m)$ implies in particular that none of the eigenvalues of a Poincare map $P_{c}$ is a $m$-th root of unity. Clearly, $(\alpha)$ is the intersection of the properties $(\alpha, m), m>0$. Therefore it suffices to show that $(\alpha, m)$ is residual, i.e., that it contains the intersection of a countable number of open dense subsets.

Consider, for each real $t>0$, the property:

$(\alpha, m, t)$ The property $(\alpha, m)$ does hold for all a belonging to Poincaré maps $P_{c}$ where the geodesic $c$ has length $\leq t$.

To prove that $(\alpha, m)$ is residual we refer to Theorem 1 in [10]. It is enough to show that

(i) $(\alpha, m, t)$ is dense, and

(ii) $(\alpha, m, t)$ is contained in the interior of $\left(\alpha, m, \frac{1}{2} t\right)$.

Indeed, if this is known it follows that each $\left(\alpha, m, \frac{1}{2} t\right)$ contains a subset which is open, dense and hence the intersection of these sets over all $t=1,2,3, \cdots$, or equivalently that $(\alpha, m)$ contains the intersection of a countable family of open dense sets; cf. [10].

To see that (i) holds we first observe that the set of $g \in \mathscr{G}$ where all geodesics are nondegenerate ("bumpy metric" of Abraham [1]) is dense. Then there are only finitely many closed geodesics of length $\leq t$. An appropriate small modification of the metric will suffice to ensure that $(\alpha, m, t)$ holds.

The validity of (ii) uses the fact that a sufficiently small perturbation of a metric $g$ satisfying $(\alpha, m, t)$ will carry closed geodesics $c$ of length $\leq \frac{1}{2} t$ into closed geodesics of length $\leq \frac{1}{2} t+\varepsilon$ such that the Poincaré maps of these closed geodesics still satisfy $(\alpha, m)$; this follows from the fact that the property $(\alpha, m)$ for the exponents $a$ of the eigenvalues $e^{ \pm 2 \pi i a}$ of the Poincare map $P_{c}$ defines an open invariant (under conjugation) subset in the space of all symplectic maps.

2. In this section we give several results which will be used later.

2.1. Given a (nontrivial) closed geodesic $c=c(t), 0 \leq t \leq \omega$, let $c^{m}=$ $c(t), 0 \leq t \leq m \omega, m$ a positive integer, be the $m$-fold covering of $c$. According to Bott ([2], see also [9]), associated with $c$ there is a function $I_{c}(\quad): S^{1}$ $\rightarrow N$ on the unit circle. The value $I_{c}(\rho), \rho \in C,|\rho|=1$, is called the $\rho$-index of $c$. The index of $c^{m}$ is then given by 


$$
\text { index } c^{m}=\sum_{\rho^{m=1}} I_{c}(\rho) .
$$

We are also interested in the average index of $c$ :

$$
\bar{I}_{c}:=\lim _{m \rightarrow \infty} \frac{1}{m} \text { index } c^{m} \text {. }
$$

Denote by $\left\{\rho_{j}, \bar{\rho}_{j}\right\}$ the set of roots of modulus 1 of the Poincare map $P_{c}$ associated with $c$, and put

$$
\rho_{j}=e^{2 \pi i a_{j}} \quad \text { with } 0 \leq a_{j} \leq \frac{1}{2} .
$$

If none of the $\rho_{j}$ is a root of unity, as it is the case under the hypothesis $(\alpha)$, we have $0<a_{j}<\frac{1}{2}$ and therefore can assume

$$
0<a_{1}<\ldots<a_{l-1}<\frac{1}{2} .
$$

We also define $a_{0}=0$ and $a_{l}=\frac{1}{2}$. The function $I_{c}(\quad)$ is constant on the connected components of $S^{1}-\left\{\rho_{j}, \bar{\rho}_{j}\right\}$. Moreover, $I_{c}\left(\rho_{j}\right) \leq I_{c}(\rho)$ for $\rho$ near $\rho_{j}$. If we denote by $I_{c, j}$ the value of $I_{c}()$ on the arc $\rho=e^{2 \pi i a}, a_{j-1}<a<a_{j}$, then for $\bar{I}_{c}$ we have the formula

$$
\bar{I}_{c}=2 \sum_{j=1}^{l}\left(a_{j}-a_{j-1}\right) I_{c, j} .
$$

In particular, if $l-1=0, I_{c, j}=$ const. $=\bar{I}_{c}$. If index $c^{m}=0$ not for all $m$, then not all $I_{c, j}=0$. Hence in this case there exist numbers $A_{c}, a_{c}>0$, and $b_{c}$ such that $m A_{c} \geq$ index $c^{m}>m a_{c}+b_{c}$.

2.2. Recall that associated with the compact simply connected riemannian manifold $M$ we have the Hilbert manifold $\Lambda M$ of $H^{1}$-maps $c: S^{1} \rightarrow M$; cf. [3], [5], [7]. On $\Lambda M$ we are given a riemannian metric of which we denote the scalar product by $\langle,\rangle_{1}$.

Moreover, we have a differentiable function $E: \Lambda M \rightarrow R$ on $\Lambda M$, the energy integral

$$
c \mapsto \int_{S^{1}}\langle\dot{c}, \dot{c}\rangle(t) d t
$$

such that the critical points of $E$ are precisely the nontrivial closed geodesics and the constant maps

$$
c_{p}: S^{1} \rightarrow M \quad \text { with } c_{p}(t)=p .
$$

The Hilbert manifold $\Lambda M$ has the same homotopy type as the space of continuous maps

$$
c: S^{1} \rightarrow M
$$


with the compact open topology.

$\Lambda M$ is the total space of a fibration over $M$ where the projection $\pi$ is given by associating with $c=c(t)$ the initial equal end point. The fibre over $p \in M$ consists of the loop space $\Omega M$ based at $p$, and the constant maps define a section :

$$
p \in M \longmapsto c_{p} \in \Lambda M \stackrel{\pi}{\longmapsto} p \in M .
$$

Sullivan has determined the (minimal) model of $A M$; cf. [11]: The generators of the model will be the generators $y$ of the model of $M$ and the generators $\bar{y}$ of the model of $\Omega M$, where $\bar{y}$ corresponds in the canonical manner to $y$, and $\operatorname{dim} \bar{y}=\operatorname{dim} y-1$. The differential $d y$ is the same as in the model of $M$ whereas $d \bar{y}$ is defined as $\overline{d y}$; here the bar is extended to polynomials in the generators as a right derivation.

Now let $x$ be an odd dimensional generator of the model of $M$ of lowest dimension. $d x$ then is a polynomial in even dimensional generators $e_{1}, \cdots, e_{l}$. By

$$
w_{r}:=\bar{e}_{1} \wedge \cdots \wedge \bar{e}_{l} \wedge \bar{x}^{r}
$$

we can obtain a sequence of nontrivial elements in the rational homology of $\Lambda M$ with $\left\{\operatorname{dim} w_{r}\right\}$ forming an arithmetic sequence. The proof of this is an easy consequence of the previous results-note that $d \bar{e}_{j}=0$ and that $\bar{e}_{j}^{2}=0$ and that the boundaries are contained in the ideal generated by the generators of the model of $M$.

Since the elements $w_{r}$ all belong to the cohomology of $\Omega M \subset \Lambda M$ we can represent the corresponding dual homology classes, which we also denote by $w_{r}$, by cycles lying in a fibre $\Omega M$ of $\Lambda M$. To give a more precise description of these cycles we observe that each generator $y$ of the model of $M$ can be represented by a homotopy class

$$
y: S^{\operatorname{dim} y} \rightarrow M .
$$

Associated with $y$ in a canonical way there is a homotopy class

$$
\bar{y}: S^{\operatorname{dim} \bar{y}} \rightarrow \Omega M,
$$

where $S^{\mathrm{dim} g}$ can be represented by the family of circles passing through the base point of $S^{\mathrm{dim} \tilde{y}}$ and orthogonal to a given sphere of codimension 1 on $S^{\text {dim } \bar{y}}$, containing the base point.

In the case of the Sullivan class $w_{r}$ we consider the product of spheres with base point :

$$
S^{\mathrm{dim} e_{1}} \times \cdots \times S^{\mathrm{dim} e_{l}} \times S^{\mathrm{dim} x} \times \cdots \times S^{\mathrm{dim} x} .
$$

For each of these spheres we have maps $e_{1}, \cdots, e_{l}, x, \cdots, x$ into $M$, as above. 
Now for each of these spheres take a representation of $S^{\text {dim } \vec{e}_{1}}, \ldots$, etc. by circles. Forming the product of these circles (in the sense of loops) on the factors of (1) we get a $\operatorname{dim} w_{r}$-parameter family of closed curves which are mapped via the product of the maps $e_{1}, \cdots, x$ into $M$. This is a cycle of $\Lambda M$, represented by a cycle of $\Omega M$.

2.3. Finally, in this section we want to recollect some properties of the Morse theory of the negative gradient flow of the differentiable function $E$ on $\Lambda M$; cf. [5], [7], and Eliasson [3], [4].

First of all, $-\operatorname{grad} E$ satisfies the condition (C) of Palais and Smale. Since $E \geq 0$, the flow

$$
\varphi_{s}: \Lambda M \rightarrow \Lambda M
$$

determined by the vector field $-\operatorname{grad} E$ is defined for all $s \geq 0$ and every orbit $\varphi_{s} c^{\prime}, s \geq 0$, contains a critical point of $E$ (i.e., a constant curve or a nontrivial closed geodesic) in its limit set.

Moreover, on $\Lambda=\Lambda M$ we have the continuous canonical $S^{1}$-action

$$
\begin{aligned}
S^{1} \times \Lambda M & \mapsto \Lambda M, \\
\left(z=e^{2 \pi i a}, c=c(t)\right) & \mapsto z \cdot c=c(t+a),
\end{aligned}
$$

and the involution

$$
\begin{aligned}
& \vartheta: \Lambda M \rightarrow \Lambda M, \\
& c=c(t) \mapsto \vartheta c=c(1-t) .
\end{aligned}
$$

Both operations are isometries and leave $E$ invariant. Hence they commute with the negative gradient flow. In particular, if $c$ is a critical point so are the elements $z \cdot c$ and $\vartheta c$.

Assume now that all critical orbits $S^{1} \cdot c, c \neq$ const., are one-dimensional nondegenerate critical submanifolds. This is equivalent to the assumption that none of the Poincaré maps $P_{c}$ of a closed geodesic $c$ has 1 as eigenvalue; cf. [8]. In particular, it is satisfied if the hypothesis $(\alpha)$ of $\S 1$ holds.

Under our assumption we can apply to $(\Lambda M,-\operatorname{grad} E)$ the standard results of Morse theory; actually, we will use equivariant Morse theory with respect to the $S^{1}$-action and the involution $\vartheta$.

For any real $\kappa \geq 0$ we put

$$
\Lambda^{\kappa}:=E^{-1}[0, \kappa], \quad \Lambda^{\kappa-}:=E^{-1}[0, \kappa[.
$$

In particular, $\Lambda^{0}$ consists of the constant curves. The canonical section

$$
M \rightarrow \Lambda^{0} \subset \Lambda: p \mapsto c_{p}
$$

is an isometric embedding; $\Lambda^{0}$ is a nondegenerate critical submanifold. 
Let $c$ be a critical point not in $\Lambda^{0}$, i.e., $E(c)=\kappa>0$. The tangent space $T_{c} \Lambda$ of $\Lambda=\Lambda M$ at $c$ then has an orthogonal decomposition

$$
T_{c} \Lambda=T_{c}^{-} \Lambda \oplus T_{c}^{0} \Lambda \oplus T_{c}^{+} \Lambda,
$$

which is given by the eigenspaces of the Hessian $D^{2} E(c)$ belonging to eigenvalues $<0,=0$ and $>0$, respectively. In particular, $\operatorname{dim} T_{c}^{-} \Lambda=I_{c}=\operatorname{index} c$, $\operatorname{dim} T_{c}^{0} \Lambda=1$, whereas $T_{c}^{+} \Lambda$ is an infinite dimensional Hilbert space. Corresponding to the subspaces $T_{c}^{-}=T_{c}^{-} \Lambda$ and $T_{c}^{+}=T_{c}^{+} \Lambda$ we have the immersions

$$
\begin{aligned}
& i_{c}^{-}:\left(T_{c}^{-}, 0\right) \rightarrow\left(\Lambda^{c}, c\right), \\
& i_{c}^{+}:\left(T_{c}^{+}, 0\right) \rightarrow\left(\Lambda-\Lambda^{x-}, c\right),
\end{aligned}
$$

which carry the rays through the origin into full $\varphi_{s}$-orbits having $c$ as limit set for $s \rightarrow-\infty$ and $s \rightarrow+\infty$, respectively.

We call $i_{c}^{-}$and $i_{c}^{+}$the unstable and stable manifolds through $c$, respectively. We can use a neighborhood of the origin of $T_{c} \Lambda$ as local coordinates of $\Lambda$ near $c$ such that $E$ in these coordinates is given by

$$
E(x)=-\left|x^{-}\right|^{2}+\kappa+\left|x^{+}\right|^{2},
$$

where

$$
x=\left(x^{-}, x^{0}, x^{+}\right) \in T^{-} \Lambda \oplus T^{0} \Lambda \oplus T^{+} \Lambda .
$$

Eliasson [4] has shown that every orbit $\varphi_{s} c^{\prime}$ converges, for $s \rightarrow \infty$, to a critical point, say $c$. That is to say, every orbit $\varphi_{s} c^{\prime}$ belongs to the stable manifold of a uniquely determined critical point.

Let $c$ be a nontrivial closed geodesic, and $E(c)=\kappa>0$. We denote by $D_{c}^{-}\left(S^{1}\right)$ the so called negative disc bundle over the orbit $S^{1} \cdot c$, i.e., the fibre $D_{c}^{-}(z)$ over $z \cdot c$ in the unit disc in $T_{z \cdot c}^{-} \Lambda$.

Assume for simplicity that there are no other critical points in $E^{-1}(\kappa)$ besides $z \cdot c, z \in S^{1}$, and $\vartheta c$. Then the space $\Lambda^{k+\varepsilon}$ has, for $\varepsilon>0$ sufficiently small, the subspace

$$
\Lambda^{\kappa-\varepsilon} \cup D_{c}^{-}\left(S^{1}\right) \cup D_{\vartheta_{c}}^{-}\left(S^{1}\right)
$$

as equivariant deformation retract, where $U$ stands for an equivariant attaching of $\partial D^{-}$to $\partial \Lambda^{x-\varepsilon}$ which is determined for each fibre $D_{c}^{-}(z)$ by the unstable manifold through $z \cdot c$. Let $c \notin \Lambda^{0}$. Then the isotropy group under the $S^{1}$-action, i.e., the group $\left\{z \in S^{1} ; z \cdot c=c\right\}$, is a finite cyclic group, say $Z_{m}=\left\{e^{2 \pi i l / m}, l\right.$ $=0, \cdots, m-1\}$. We call the order $m$ of $Z_{m}$ the multiplicity of $c$. In particular, if $m$ is the multiplicity of a nontrivial closed geodesic $c$, then $c$ is the $m$ fold covering $c_{0}^{m}$ of a prime closed geodesic $c_{0}$.

Let $c$ be a closed geodesic, $E(c)=\kappa>0$, and index $c=I_{c}$. Assume that 
the negative disc is a nontrivial homology class (with rational coefficients) of $\left(\Lambda, \Lambda^{*-}\right)$, i.e.,

$$
\left(D^{-}(c), \partial D^{-}(c)\right) \rightarrow\left(\Lambda, \Lambda^{x-}\right)
$$

defines an $I_{c}$-dimensional element $\neq 0$ of $H_{*}\left(\Lambda, \Lambda^{\kappa-}\right)$. Then also $\left(D^{-}(\vartheta c)\right.$, $\left.\partial D^{-}(\vartheta c)\right)$ defines such a nontrivial element. Assume that these elements are (up to the sign) homologous. That means that there is a chain $v$ in $\left(\Lambda, \Lambda^{-}\right)$ such that

$$
\partial v=D^{-}(c) \mp D^{-}(\vartheta c) .
$$

In this equation we can replace $v$ by $\varphi_{s} v$, for all $s \geq 0$. Since, for $\varepsilon>0$ sufficiently small, $\Lambda^{x+\varepsilon}$ is being deformed by $\varphi_{s}$ into

$$
\Lambda^{\kappa-\varepsilon} \cup \bigcup_{c^{\prime}}\left(D_{c^{\prime}}^{-}\left(S^{1}\right) \cup D_{\vartheta c^{\prime}}^{-}\left(S^{1}\right)\right)
$$

where $c^{\prime}$ runs through representatives of the finitely many critical $\left(S^{1}, \vartheta\right)$-orbits in $E^{-1}(\kappa)$, it follows that the chains $v$ satisfying (2) have a critical value $\kappa^{*}>\kappa$ :

$$
\kappa^{*}=\inf _{v} \sup _{c \in v} E(c) \text {. }
$$

That is to say, there is a critical element $c^{*}, E\left(c^{*}\right)=\kappa^{*}$, such that the unstable manifold

$$
i_{c^{*}}^{-}:\left(T_{c^{*}}^{-}, 0\right) \rightarrow\left(\Lambda, c^{*}\right)
$$

contains $c$ in its limit set. Moreover,

$$
I_{c^{*}}=\operatorname{dim} T_{c^{*}}^{-}=I_{c}+1=\operatorname{dim} T_{c}^{-}+1 .
$$

Since these relations are equivariant with respect to the $S^{1}$-action, it follows that the isotropy group $\boldsymbol{Z}_{m *}$ of $c^{*}$ must operate as identity on $c$, i.e., the multiplicity $m^{*}$ of $c^{*}$ divides the multiplicity $m$ of $c$.

3. We now come to the main result of our paper:

3.1. Theorem. Let $M$ be a compact simply connected differentiable manifold. Denote by $\mathscr{G}^{*}$ the residual subset of the space $\mathscr{G}=\mathscr{G}(M)$ of riemannian metrics satisfying the condition $(\alpha)$ of $\S 1$. Then for every $g^{*} \in \mathscr{G}^{*}$ the riemannian manifold $\left(M, g^{*}\right)$ has an infinite number of prime closed geodesics.

Remark. Briefly we therefore can say that generically a riemannian manifold with finite fundamental group has an infinite number of prime closed geodesics.

Proof. We assume that there are only finitely many prime closed geodesics on $\left(M, g^{*}\right)$, and we are going to derive a contradiction from this hypothesis.

First of all it follows that there is one prime closed geodesic, say $c$, such 
that an infinite number of the Sullivan classes $w_{r}$, (cf. $\S 2.2$ ), has coverings $c^{m}$ of $c$ as critical point. More precisely, there are sequences $\left\{r_{i}\right\}$ and $\left\{m_{i}\right\}$ in $N$ such that $w_{r_{i}}$ has $c^{m_{i}}$ as critical point. Note that since each $w_{r}$ has a representative as a connected singular cell of dimension $\operatorname{dim} w_{r}$ in $\Omega M, w_{r_{i}}$ is neither $S^{1}$-invariant and nor $\vartheta$-invariant. This means that $w_{r_{i}}$ near $c^{m_{i}}$ is representable as the negative disc $D^{-}\left(c^{m_{i}}\right)$, whereas $\vartheta D^{-}\left(c^{m_{i}}\right)=D^{-}\left(\vartheta c^{m_{i}}\right)$ does not occur in the representative of $w_{r_{i}}$.

On the other hand, one knows that $\vartheta$ operates on the generators

$$
\bar{y}: S^{\operatorname{dim} y} \rightarrow \Omega M
$$

of the model of $\Omega M$ by multiplication with -1 ; this is immediate from the definitions. Hence $\vartheta w_{r}= \pm w_{r}$, and the local nontrivial cycle $D^{-}\left(c^{m_{i}}\right)$ is homologous to the local nontrivial cycle $\pm D^{-}\left(\vartheta c^{m_{i}}\right)$ in $\left(\Lambda, \Lambda^{\kappa_{i}-}\right)$, where $\kappa_{i}:=E\left(c^{m_{i}}\right)$ $=m_{i}^{2} E(c)$. From $\S 2.3$ we therefore have the existence of a critical point $c^{*}(i)$ with

$$
\partial D^{-}\left(c^{*}(i)\right) \supset D^{-}\left(c^{m_{i}}\right)
$$

Under our hypothesis we can assume that all these $c^{*}(i)$ are coverings of the same prime closed geodesic $c^{*}$, i.e., that there exists a sequence $\left\{m_{i}^{*}\right\}$ in $N$ such that $c^{*}(i)=c^{* m_{i}^{*}}$. Hence we have, from $\S 2.3$,

$$
\text { index } c^{* m_{i}^{*}}=\operatorname{index} c^{m_{i}}+1 \text {. }
$$

Moreover, $m_{i}^{*}$ divides $m_{i}$, i.e., for each $i$ there is a positive integer $q_{i}$ such that $m_{i}=q_{i} m_{i}^{*}$. We claim that these $q_{i}$ must be bounded. Indeed, from (3) and the last formula in $\S 2.1$ we have the relation

$$
m_{i}^{*} A_{c^{*}} \geq \operatorname{index} c^{* m_{i}^{*}} \geq q_{i} m_{i}^{*} a_{c}+b_{c}+1 \quad \text { with } a_{c}>0 .
$$

Hence $q_{i}$ is bounded, and we can assume $q_{i}=q=$ const. $\geq 1$ for all $i$.

Using (3) and the formulas for the average indices of $c^{*}$ and $c$ in $\S 2.1$ we thus get the relation:

$$
\sum_{j=1}^{l^{*}}\left(a_{j}^{*}-a_{j-1}^{*}\right) I_{c^{*}, j}=q \sum_{j=1}^{l}\left(a_{j}-a_{j-1}\right) I_{c, j} .
$$

Since the $a_{j}^{*}, a_{j}$ are free over the rationals, we have

$$
I_{c^{*}, j}=\bar{I}_{c^{*}}, \quad I_{c, j}=\bar{I}_{c},
$$

and therefore, in consequence of (3) again,

$$
m_{i}^{*} \bar{I}_{c^{*}}=q m_{i}^{*} \bar{I}_{c}+1
$$


for a full sequence $\left\{m_{i}^{*}\right\}$. (4) is the desired contradiction, and we thus have proved our theorem.

4. In the connection with our Theorem 3.1 we still want to prove another result which, together with the fix point theorem of Birkhoff and Lewis, will give a different proof of Theorem 3.1 ; cf. [8].

4.1. Lemma. Let $M$ be a compact differentiable manifold with finite fundamental group, and assume that $g$ is a riemannian metric on $M$ such that all closed geodesics are hyperbolic. Then $(M, g)$ has an infinite number of prime closed geodesics.

Remarks. Recall that a hyperbolic closed geodesic is nondegenerate. It seems unlikely that on a compact differentiable manifold with finite fundamental group there can exist a riemannian metric $g$ such that all closed geodesics on $(M, g)$ are hyperbolic.

In any case, from Lemma 4.1 it follows that if there would be only a finite number of prime closed geodesics, then there must be one which is nonhyperbolic. Generically, near such a geodesic there is an infinite number of other prime closed geodesics; cf. [8], [10].

Proof of Lemma 4.1. We simply observe that under the assumptions of Lemma 4.1 and the hypothesis that there are only finitely many prime closed geodesics, we get a relation of type (3). Assume that all these geodesics are hyperbolic, i.e., all $a_{j}=0$ for $j<l$ and $a_{j}^{*}=0$ for $j<l^{*}$. Then (3) implies (4), a contradiction.

Added in proof. Since this paper was written, the existence of infinitely many closed geodesics without any restrictions on the riemannian metric has been proved; see W. Klingenberg, Lectures on closed geodesics, Manuscript, University of Bonn, 1976.

\section{References}

[1] R. Abraham, Bumpy metrics, Global Analysis (Proc. Sympos. Pure Math. Vol. XIV, Berkeley, Calif., 1968), Amer. Math. Soc. 1970, 1-3.

[2] R. Bott, On the iteration of closed geodesics and the Sturm intersection theory, Comm. Pure Appl. Math. 9 (1956) 171-206.

[3] H. Eliasson, Morse theory for closed curves, Sympos. on infinite dimensional topology, Annals of Math. Studies, No. 69, Princeton University Press, Princeton, 1972, 63-77.

[4] - Convergence of gradient curves on Hilbert manifolds, Math. Z. 136 (1974) 107-116.

[ 5] P. Flaschel \& W. Klingenberg, Riemannsche Hilbertmannigfaltigkeiten. Periodische Geodätische, Lecture Notes in Math. Vol. 282, Springer, Berlin, 1972.

[6] D. Gromoll \& W. Meyer, Periodic geodesics on compact riemannian manifolds, J. Differential Geometry 3 (1969) 493-510.

[ 7 ] W. Klingenberg, Closed geodesics, Ann. of Math (2) 89 (1969) 68-91.

[ 8 ] Closed geodesics on riemannian manifolds, Proc. 13th Biennial Sem. Canad. Math. Congress, Vol. 1, 1972, 69-92.

[9] - Der Indexsatz für geschlossene Geodätische, Math. Z. 139 (1974) 231-256.

[10] W. Klingenberg \& F. Takens, Genetic properties of geodesic flows, Math. Ann. 197 (1972) 323-334. 
[11] D. Sullivan, Topology of manifolds and differential forms, Manifolds-Tokyo 1973 (Proc. Internat. Conf. on Manifolds and Related Topics in Topology), University of Tokyo Press, Tokyo, 1975, 37-49.

UNIVERSITY OF BONN 